\title{
Physico-chemical tools: towards a detailed understanding of the architecture of targeted radiotherapy nanoparticles
}

Gema Cabello $^{\mathrm{a}, *}$, Kenneth C Nwoko ${ }^{\mathrm{b}}$, Marco Mingarelli ${ }^{\mathrm{a}}$, Abbie C McLaughlin ${ }^{\mathrm{b}}$, Laurent Trembleau ${ }^{\mathrm{b}}$, Joerg Feldmann ${ }^{\mathrm{b}}$, Angel Cuesta ${ }^{\mathrm{b}}$, Tim AD Smith,

${ }^{a}$ School of Medicine, Medical Sciences and Nutrition, University of Aberdeen, Foresterhill, Aberdeen AB25 2ZD, UK.

${ }^{\mathrm{b}}$ Department of Chemistry, School of Natural and Computing Sciences, University of Aberdeen, Aberdeen AB24 3UE, UK.

Address for correspondence:

Dr Tim Smith (t.smith@abdn.ac.uk); Dr Gema Cabello (gema.cabellocarramolino@abdn.ac.uk)

Biomedical Physics Building, University of Aberdeen, Foresterhill, Aberdeen AB25 2ZD, UK

\begin{abstract}
Targeted radiotherapy is proving to be an effective alternative to external beam radiotherapy for cancer treatment. Gold nanoparticles are bio-compatible, commercially available and readily functionalised, which makes them perfect candidates for the delivery of cytotoxic radionuclides labelled with antibodies to proteins abnormally expressed on cancer tissue. However, there is a lack of information regarding the efficacy of the successive modification steps involved in the functionalisation process, as well as of the actual final state of the nanoparticles prior to preclinical tests, which results in a very inefficient screening and that will further impact on biological barriers, such as half-life interactions with serum proteins. Here, gold nanoparticles (15 nm diameter) were functionalised with linkers for antibody and radionuclide conjugation, following a well-stablished method. Successful coating of the gold nanoparticles was demonstrated using state-of-the-art physico-chemical techniques, which include TEM, AF4-UV-ICPMS-MALS, Raman spectroscopy and force-distance spectroscopy, which have led to an accurate description of the hydrodynamic diameter of the functionalized NPs and also about the adhesion energy and elastic properties of the modified NPs. Successive steps involved in the coating led to an organic shell of $12 \mathrm{~nm}$ diameter and no nanoparticle aggregation was observed. This may be a consequence of a decrease (or even the total absence) of water adsorption on the metal surface and/or of the organic labelling, that decreases the surface tension of the particles as estimated from the atomic force microscopy force-distance curves. Radiolabelling of gold nanoparticles pre-screened using these physico-chemical tools with ${ }^{177} \mathrm{Lu}$ resulted in $>75 \%$ efficiency.
\end{abstract}

\section{Keywords}

Targeted radiotherapy, radionuclide carriers, force-distance spectroscopy, asymmetric flow field-flow fractionation, nanoparticle adhesion energy 


\section{Introduction}

Radiotherapy is highly effective at sterilising cancer stem cells, ${ }^{1}$ which represent about 1 in 1000 cancer cells in a malignancy and are responsible for tumour repopulation, development and recurrence. ${ }^{2}$ Radiotherapy induces cellular macromolecular damage e.g. to DNA by interacting either directly with the macromolecule or by producing free radicals, such as hydroxyl radicals formed from water, which react with and induce chemical changes in the macromolecule. The lethal effect of radiotherapy is generally considered to result from DNA damage. Irradiation of $\mathrm{Au}$ nanoparticles (NPs) can induce inner shell ionisations of the $\mathrm{Au}$ atoms and subsequent emission of Auger electrons, which enhance the radiation cytotoxic effect ${ }^{3}$. Consequently, Au NPs, which demonstrate selective retention by cancer cells, have been employed as radio-sensitizers in radiotherapy. ${ }^{3}$

More recently, Au NPs have been used as radiation delivery systems by surface modification with chelators bearing $\beta$-emitting radionuclides and targeted to cell surface receptors using a range of targeting moieties. ${ }^{6,12,13}$ These include antibodies to the epidermal growth factor receptor (EGFR), which is overexpressed in several cancer types including the treatmentrefractory triple-negative breast cancer. This cancer treatment approach is known as targeted radiotherapy (TR), can be considered an example of precision medicine and unlike external beam radiotherapy, it delivers a radiation dose to the primary tumour and secondary metastatic spread which similarly over-express cell surface proteins. Low-energy $\beta$-particle emitters, such as ${ }^{177} \mathrm{Lu}$, deliver their radiation dose within a short-range $(1-2 \mathrm{~mm})$ so, when targeted to cancer cells, will deposit most of their dose within the tumour, sparing surrounding non-tumour tissues. There are many types of biocompatible nanomaterials that can be modified to carry radionuclides, such as dendrimers, ${ }^{4-5}$ but are also heat labile and cannot withstand the high radiolabelling temperatures required for chelation of ${ }^{177} \mathrm{Lu}$. $\mathrm{Au}$ NPs are non-toxic, biocompatible and can be readily functionalized using bifunctional PEG linkers with thiol groups at one end that readily bind to Au producing highly stable Au-S bonds. The other end of the linker can be modified by conjugation with a chelator or with a chemical group that can be used to conjugate with a targeting moiety e.g. SVA (succinimidyl valeric acid), which is readily reacted with amino groups present in antibodies (AB). The 1,4,7,10tetraazacyclododecane-1,4,7,10-tetraacetic acid (DOTA)-type chelating agents have been extensively used, since they show high labelling efficiency with ${ }^{177} \mathrm{Lu}$ and high complex stability ${ }^{6-8}$ which has been demonstrated to play an important role in the performance of the conjugates. ${ }^{7-8}$ Further, since cytotoxic effects of these compounds are dependent on the amount of radioactivity they carry, an approach that optimises the radiolabelling efficiency also leading to a better understanding of the chemical nature of functionalised Au NPs is crucial to progress this approach to cancer treatment. A detailed characterization of all steps involved in the functionalization process is a remaining goal to ensure compound optimisation, aimed at overcoming associated obstacles related to toxicity ${ }^{9}$, dose control and tumour penetration in poorly vascularized regions as well as simplifying the labelling process.

Within this aim, we have methodically examined the assembly of Au NPs, based on a modification of a previously described radiolabelling method. ${ }^{10-12}$ The modifications, include the use of smaller, $15 \mathrm{~nm}$ Au NPs as carriers of ${ }^{177}$ Lu tagged with an anti-EGFR antibody (AB) as the targeting moiety that can bind to EGFR-overexpressing primary and metastatic cancer cells. A further modification is the radio-labelling of the chelator after attachment to the Au NPs, which is considered to be advantageous ${ }^{7}$ over pre-Au NP-conjugation radiolabelling. Using state-of-the-art physico-chemical techniques, we have obtained an accurate description of: (i) the size of fully-functionalised particles, using multidetector analysis asymmetrical flow field-flow fractionation (AF4); and (ii) the adhesion energy and elastic properties of the modified NPs, by force distance spectroscopy (FDS) and atomic force microscopy (AFM) imaging. Particle size is a critical parameter for nanostructured materials for biomedical 
applications. Multidetector-coupled AF4 has arisen as a promising technique for this purpose ${ }^{13-}$ ${ }^{17}$, where other routine characterization techniques, such as scanning and transmission electron microscopy (SEM and TEM, respectively) and dynamic light scattering (DLS), fail. ${ }^{18-21}$ Unfractionated DLS, at best, differentiates particles that vary in size by a factor of 3 to 5 , whereas AF4-MALS is capable of resolving accurately particles that vary in size by just a few percent, and additionally helps in the determination of shape. MALS detector is unaffected by aggregation, adsorption and sedimentation of particles, which are limitations associated with DLS. It also offers a vertical flow cell where the sample can pass through easily without any obstacles in its way. While DLS measures at a single scattering angle of $173^{\circ}$, MALS detector employs 21 angles, from $7^{\circ}$ to $164^{\circ}$, ensuring accuracy and precision in particle size determination when measuring complex particular samples. In addition, MALS detector can measure NP radius of gyration in the range of $8 \mathrm{~nm}$ to $500 \mathrm{~nm}$, depending on the sample.

Besides, AFM in the imaging mode has been extensively used for characterisation of nanoparticle shape and/size $\mathrm{s}^{22-24}$ and, in recent years, its application in biological samples has exponentially increased due to the development of single-molecule force spectroscopy with chemically modified AFM probes. ${ }^{25-28}$ However, despite the versatility of this technique, little attention has been paid to the characterization of nanoparticles adhesion energy by FDS. ${ }^{29-32}$ Accordingly, we have examined the NPs adhesion energy in every step of the coating process, which has led us to elaborate a construction sequence for direct radiolabelling.

To the best of our knowledge, the combination of these physico-chemical techniques has not been used before, even though they have shown to provide reliable information of the sequential functionalisation of NPs. Our experiments integrate knowledge in the areas of materials, physics, bioscience, and chemistry for the adequate characterization of radionuclide carriers in order to improve cell uptake. The provided information is suggested to lead to the development of more efficient architectures of tagged NPs for radio- and chemotherapy treatments.

\section{Materials and methods}

\subsection{Reagents and materials}

Ortho-pyridyl disulphide-polyethyleneglycol-succinimidyl valeric acid (OPSS-PEG-SVA, MW 5000) and OPSS-PEG3500-amine were purchased from Laysan Bio (AL, USA) and JemKem Technology (TX, USA), respectively. Non-coated gold NPs $(15 \mathrm{~nm})$ in $0.1 \mathrm{mM}$ phosphate buffered saline (PBS) were purchased from Stratech (Suffolk, UK). Erbitux (Merck Serono Ltd) was purchased from AAH Pharmaceuticals (UK) and a weighed amount (typically $10 \mathrm{mg}$ of lyophilisate dissolved in $0.5 \mathrm{~mL}$ of $\mathrm{H}_{2} \mathrm{O}$ ) purified on a centrifugal filter (SigmaAldrich, UK) with a $30 \mathrm{KDa}$ cut off. The concentration of Erbitux recovered from the filter was determined using the extinction coefficient of 1.4 for $1 \mathrm{mg} \mathrm{mL}^{-1}$ at $280 \mathrm{~nm}$ after measuring absorbance at $280 \mathrm{~nm}$ using a spectrophotometer (Thermo Spectronic Helios $\gamma$ ). $\left[{ }^{177} \mathrm{Lu}\right] \mathrm{LuCl}_{3}$ was purchased from Perkin-Elmer (Beaconsfield, UK) in doses of $370 \mathrm{MBq}$. All other chemicals were purchased from Sigma-Aldrich (Poole, UK) unless otherwise stated.

\subsection{Experimental}

Preparation of OPSS-PEG-DOTA. Linkers with the OPSS (Ortho-pyridyl disulphide) functionality are commonly utilised to provide binding to gold surfaces exploiting the strength of the Au-S bond. ${ }^{33}$ PEG (polyethylene glycol) coating of particle surfaces serves to enable evasion of the immune system by preventing their opsonisation (surface tagging of foreign substances in the body to expedite their removal by phagocytic cells). ${ }^{34-36}$ Surface coating is 
also essential in preventing NP aggregation. DOTA is a strong chelator of Lu facilitating the linking of ${ }^{177} \mathrm{Lu}$ to the NP. ${ }^{10,37}$

$100 \mathrm{mg}$ of DOTA was dissolved in $0.625 \mathrm{~mL}$ of $5 \mathrm{M} \mathrm{NaOH}$. The $\mathrm{pH}$ was adjusted to 8.5 by addition of $\mathrm{HCl} .15 \mathrm{mg}$ of 4-(4,6-dimethoxy-1,3,5-triazin-2-yl)-4-methyl morpholinium chloride (DMTMM) were dissolved in $100 \mu \mathrm{L}$ of $\mathrm{H}_{2} \mathrm{O}$ and mixed for $5 \mathrm{~min}$, followed by addition of $30 \mathrm{mg}$ OPSS-PEG-NH $\mathrm{N}_{2}$ dissolved in $100 \mu \mathrm{L}$ of $\mathrm{H}_{2} \mathrm{O}$ for $1 \mathrm{~h}$. The mixture was then placed in a $1 \mathrm{kDa}$ cut off dialysis capsule (Sigma-Aldrich) and the capsule was placed in 200 $\mathrm{mL}$ of water, which was changed 3 times daily for 3 days. The volume capsule was then removed from the water and the contents dried by placing them in a stream of air. The OPSSPEG-DOTA was then suspended in $\mathrm{H}_{2} \mathrm{O}$ or $\mathrm{D}_{2} \mathrm{O}$ for ${ }^{1} \mathrm{H}-\mathrm{NMR}$ analysis.

Functionalisation of Au NPs with linkers. $1 \mathrm{~mL}$ of Au NPs (containing ca. $1.64 \times 10^{12}$ particles) were centrifuged at $20000 \mathrm{~g}$ for $30 \mathrm{~min}$ and all but about $25 \mu \mathrm{L}$ the supernatant removed. OPSS-PEG-SVA $(20 \mu \mathrm{g})$ and OPSS-PEG-DOTA $(50 \mu \mathrm{g})$ were premixed in a total volume of $10 \mu \mathrm{L}$, mixed with the concentrated NPs and left overnight at room temperature, after which 10 $\mu \mathrm{g}$ of PEG-thiol (PEG-SH) was added and incubated for a further $1 \mathrm{~h}$. After addition of $1 \mathrm{~mL}$ of $\mathrm{H}_{2} \mathrm{O}$, the functionalised NPs were centrifuged at $20000 \mathrm{~g}$ for $30 \mathrm{~min}$. This was repeated to remove all non-bound linkers and the NPs suspended in $25 \mu \mathrm{L}$ of $\mathrm{H}_{2} \mathrm{O}$.

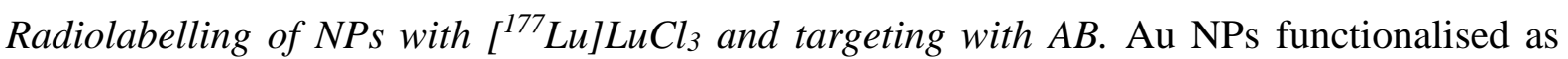
described above were mixed with $10 \mu \mathrm{L}$ of $0.5 \mathrm{M}$ citrate buffer and $20 \mathrm{MBq}$ of $\left[{ }^{177} \mathrm{Lu}\right] \mathrm{LuCl}_{3}$ then placed in a heating block and heated at $80{ }^{\circ} \mathrm{C}$ for $20 \mathrm{~min}$. Non-chelated $\left[{ }^{177} \mathrm{Lu}\right] \mathrm{LuCl}_{3}$ was removed by centrifugation after addition of $1 \mathrm{~mL}$ of $\mathrm{H}_{2} \mathrm{O}$. Labelling efficiency was determined by measuring activity in the washes and in the washed NPs by scintillation counting of diluted (1:2000) samples. The radiolabelled NPs were then suspended in $50 \mu \mathrm{L}$ of $0.1 \mathrm{M} \mathrm{NaHCO}$ containing $200 \mu \mathrm{g}$ of Erbitux and incubated overnight at room temperature. The radiolabelled and Erbitux-tagged NPs (which from now on we will design as targeted ${ }^{177} \mathrm{Lu}$-AB-labelled NPs) were suspended in $1 \mathrm{~mL}$ of PBS and centrifuged as described. This step was carried out twice and the NPs finally suspended in $100 \mu \mathrm{L}$ of PBS. They were then sterile filtered through a centrifugal $0.22 \mu \mathrm{m}$ filter by centrifuging at $4000 \mathrm{~g}$ for $2 \mathrm{~min}$. NP aggregation was not a problem but we did encounter irreversible attachment of the fully functionalised nanoparticles to the microfuge tube during the washing centrifugations after incubation with $\mathrm{AB}$. However, this was remedied by centrifuging the tagged NPs in $10 \mathrm{mM} \mathrm{NaHCO}_{3}(\mathrm{pH} 8.5)$. AB-tagged $\mathrm{Au}$ NPs functionalised with OPSS-PEG-DOTA were incubated for 20 min at $80{ }^{\circ} \mathrm{C}$ with $10 \mathrm{MBq}$ $\left[{ }^{177} \mathrm{Lu}\right] \mathrm{LuCl}_{3}$, after which $1 \mathrm{~mL}$ of water was added. NPs were then pelleted by centrifugation, washed with a further $1 \mathrm{~mL}$ of water and centrifuged again. Radioactivity in the supernatants and pellet were measured in a well counter. Radiolabelling efficiency was the activity in the washed pellet as a percentage of the total activity and achieved on average $>75 \%$. The preparation was found to be stable for $24 \mathrm{~h}$ at room temperature $(98 \%( \pm 2) \mathrm{n}=5)$ and up to 5 days $(95 \%( \pm 2) \mathrm{n}=2)$ at $4^{\circ} \mathrm{C}$.

Determination of EGFR expression. EGFR expression was determined using an ELISA assay kit (Fisher Scientific UK) following the manufacturer's (Invitrogen Thermo-Fisher Scientific) instructions.

\subsection{Instrumentation}

TEM images were acquired with a JEM-1400 Plus transmission electron microscope (JEOL, Japan) operated at $120 \mathrm{kV}$.

Asymmetric flow field flow fractionation coupled with UV absorption, multi angle light scattering (MALS) and inductively coupled plasma mass spectrometry (ICPMS) detection (AF4-UV-MALS-ICPMS) measurements were performed with an AF2000 MT FFF (Postnova 
Analytics, GmbH) and 8800 Triple Quadrupole ICP-MS/MS (Agilent, USA). The AF4 multiflow separation system was equipped with a PN7520 solvent degasser, PN5300 auto-sampler, 2 isocratic pumps (PN1150) for injection and focus flows, and two Kloehn syringe pumps for generation of cross flow. Its trapezoid separation channel (PN4020, Postnova Analytics) had dimensions of $335 \times 60 \times 40 \mathrm{~mm}$, and internal thickness of $350 \mu \mathrm{m}$, fitted with a regenerated cellulose acetate filter membrane (MWCO $=10 \mathrm{kDa}$, Postnova Analytics, GmbH). The UV/vis-detector (PN3211) spectral range was $190 \mathrm{~nm}-700 \mathrm{~nm}$. The MALS detector had 21 scattering angles ranging from $7^{\circ}$ to $164^{\circ}$, with that of $90^{\circ}$ showing the most intense peak. The Nova AF2000 FFF Analysis software was used for the study of MALS results. A PN9050 ICPMS interface enabled online connection of the AF4 outflow to the ICPMS detector. MilliQ water (Millipore Purification System, $18 \mathrm{M} \Omega \mathrm{cm}$ ) was used as mobile phase/eluent in all cases. Sample volume injections were typically of $20 \mu \mathrm{L}$ under a $0.5 \mathrm{~mL} \mathrm{~min}^{-1}$ flow rate, and the channel was rinsed with water for 3 min between each sample injection to avoid memory effects (specific elution conditions are shown in Table S1 in the Supporting Information). ICPMS was operated with a RF power (1550 MW), $0.79 \mathrm{~L} \mathrm{~min}^{-1}$ carrier gas, $2.8 \mathrm{~mL} \mathrm{~min}^{-1} \mathrm{H}_{2}$ as collision gas and spray chamber temperature of $2{ }^{\circ} \mathrm{C}$. Data was acquired in the time resolved analysis (TRA) mode at $0.3 \mathrm{sec}$ acquisition time.

All Raman experiments were performed using an inVia High Resolution Confocal Raman Spectrometer (Renishaw plc, Wotton-under-edge, UK). The excitation line at $785 \mathrm{~nm}$ was provided by an NIR diode laser (max. $300 \mathrm{~mW}$ ) and 1200 lines/mm spectrographic grating. A 50x objective for laser illumination and scattered light collection was used with a theoretical spot diameter of $1.7 \mu \mathrm{m}$.

Atomic force microscopy (AFM) measurements were performed with a NanoObserver AFM microscope (CS Instruments, France), using silicon nitride cantilevers from APPNANO (SPM probe Fort: $f=200-400 \mathrm{kHz} ; k=13-77 \mathrm{~N} \mathrm{~m}^{-1}$ ). It is worth noting that the spring constant of the cantilever was calculated from the average dimensions of the cantilever provided by the manufacturer. Samples were deposited on muscovite mica substrates from Agar Scientific.

Ultima Gold $^{\mathrm{TM}}$ (Perkin Elmer, UK) on a Packard Tri-Carb 2100TR scintillation counter was used for measuring the activity of ${ }^{177} \mathrm{Lu}$.

\section{Results and discussion}

\subsection{NP coating characterization}

The sequence of construction of targeted NPs is shown in Fig. 1. It is similar to that reported elsewhere, ${ }^{11-12,} 37$ with several modifications. It involves: (1) mixture of OPSS-PEG-DOTA and OPSS-PEG-SVA; (2) attachment of these functional linkers to the Au NPs; (3) addition of PEG-SH to ensure complete coverage of the Au surface, followed by removal of excess linkers by centrifugation; (4) radiolabelling of the functionalised NPs with ${ }^{177} \mathrm{Lu}$, followed by removal of non-chelated ${ }^{177} \mathrm{Lu}$; (5) incubation with $\mathrm{AB}$, followed by removal of non-conjugated $\mathrm{AB}$. 


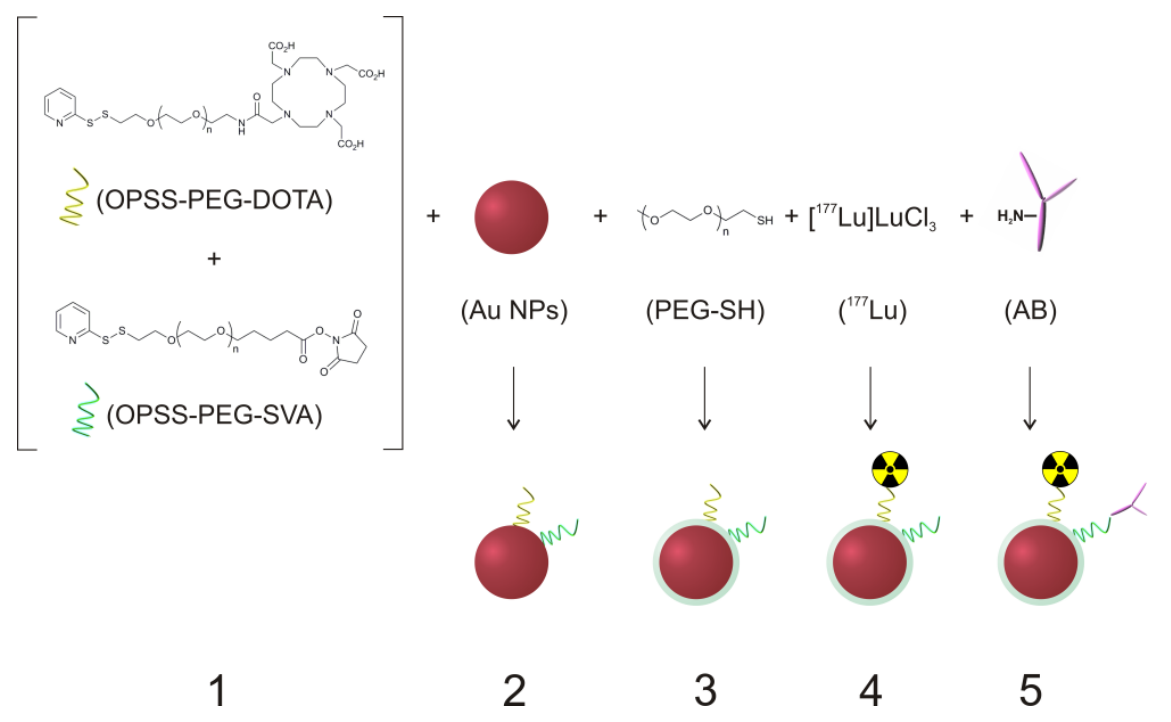

Fig. 1. Sequence of construction of tagged $15 \mathrm{~nm}$ Au NPs. (1) Mixture of functional linkers, OPSS-PEG-DOTA and OPSS-PEG-SVA; (2) attachment of the functional linkers to the Au NPs; (3) addition of PEG-SH; (4) radiolabelling of the functionalised NPs with ${ }^{177} \mathrm{Lu}$; (5) incubation with $\mathrm{AB}$.

\subsubsection{TEM}

Commercial $\mathrm{Au}$ NPs $15 \mathrm{~nm}$ in diameter showed a narrow size distribution and spherical morphology (Fig. 2A). After coating the NPs with OPSS-DOTA, OPSS-SVA-AB and PEG$\mathrm{SH}$, no significant changes in size or size dispersion were observed (Fig. 2B), which indicates that none of the processes involved in the chemical modification of the NPs provoked their aggregation. Fig. 2C shows TEM images of HCT8 cells after exposure to OPSS-DOTA-, OPSS-SVA-AB- and PEG-SH-labelled NPs for $4 \mathrm{~h}$. Targeted NPs can be found attached to the outer cell membrane, cytoplasm and structures within the cytoplasm, but not to the nucleus. This is generally the case for gold NPs unless they are specifically tagged for translocation into the nucleus. ${ }^{38}$ The absence of NPs inside the nucleus would preclude the use of therapies based on radionuclides emitting Auger electrons, which have a mean-free path of $<0.5 \mu \mathrm{m}$ which is of the order of sub-cellular dimensions, and, thus, need to be within the nucleus to cause DNA damage.
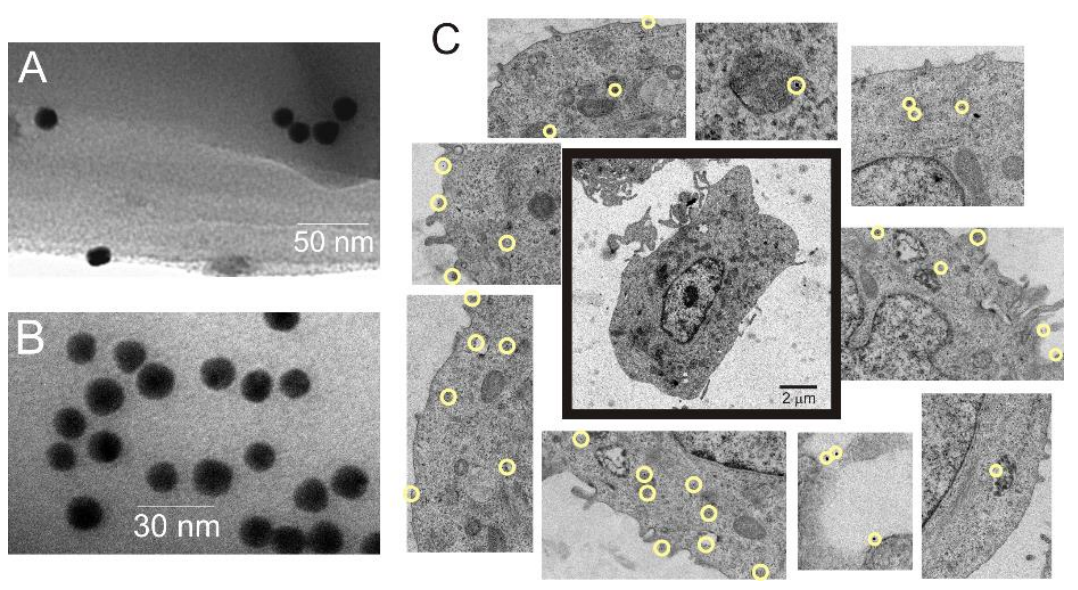
Fig. 2. TEM images of $15 \mathrm{~nm}$ gold NPs (A) and $15 \mathrm{~nm}$ gold NPs coated with OPSS-DOTA, OPSS-SVA-AB and PEG-SH (B), and HCT8 cells exposed to coated $15 \mathrm{~nm}$ Au NPs for 4 hours $(\mathrm{C})$.

\subsubsection{AF4-UV-MALS-ICPMS}

The working principle of AF4 is described in the Supporting Information and illustrated in Fig. S1. One advantage of AF4-UV-MALS-ICPMS is that the NP-organic system is characterized as an ensemble by the UV and MALS detectors, while additional online coupling of the AF4 to ICPMS enables unambiguous element specific detection. However, surface plasmon resonance of gold NPs ${ }^{39-40}$ did not allow for accurate size determination by MALS. The Nova FFF software allows for calculation of size distribution based on anisotropic scattering. However, in the case of Au NPs, isotropic scattering (evenly scattering in all directions) is observed because of their singular optical features, making the calculation of nanoparticle size distribution using the FFF software unreliable. Consequently, diameters of the NP-organic shell system were evaluated by external calibration (Fig. S2) from the UV retention times of $\mathrm{Au}$ NPs standards of $5 \mathrm{~nm}, 15 \mathrm{~nm}, 50 \mathrm{~nm}$ and $60 \mathrm{~nm}$ (Fig. 3A). Retention times obtained for gold standards as obtained from fractograms monitored with the UV absorption detector are shown in Fig. 3A. ICPMS fractograms at m/z 197 (Fig. 3B) corroborated that all bands correspond to gold, and that artefacts are absent. MALS fractograms of the gold standards were acquired at $90^{\circ}$ (Fig. S3 and S4). $5 \mathrm{~nm}$ gold NPs MALS signal was at the background level (Fig. S4), despite the intense bands found with the UV absorbance and ICPMS detectors. AF4UV-ICPMS measurements of gold NPs (15 nm diameter) with different organic coatings were also carried out (Fig. 3C and D), and the particle diameters were calculated as a function of the UV retention times using the calibration curve (Fig. S2). Diameters of coated Au NPs are shown in Table 1. Gold NPs coated only with OPSS-PEG-DOTA or OPSS-PEG-SVA showed similar diameters (42 and $41 \mathrm{~nm}$, respectively), which is in good agreement with the length of the aliphatic chains. The diameter of OPSS-PEG-SVA-functionalised NPs increased slightly after AB binding but, interestingly, the OPSS-PEG-DOTA + OPSS-PEG-SVA-AB corona contracts after addition blocking the remaining NP surface with PEG-SH. This might be due to a shrinking of the pre-existing organic shell, probably due to $\mathrm{H}$ bonds and other types of dipoledipole interactions. Once completely coated (OPSS-PEG-DOTA/SVA-AB/PEG-SH), the gold-shell system showed diameters of $40 \mathrm{~nm}$. 

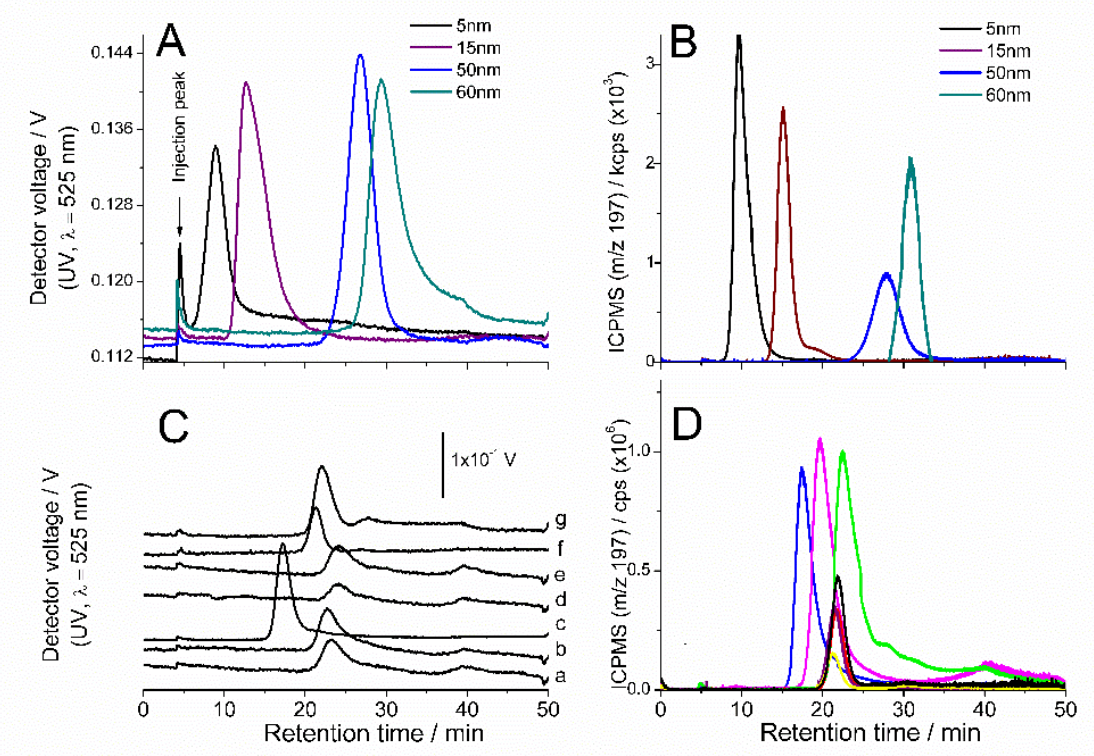

Fig. 3. (A) AF4 fractograms of $5 \mathrm{~nm}$ (black), $15 \mathrm{~nm}$ (purple), and $60 \mathrm{~nm}$ (green) commercial gold NPs, monitored with a UV detector at $525 \mathrm{~nm}$. (B) AF4 fractograms of $5 \mathrm{~nm}$ (black), 15 $\mathrm{nm}$ (purple), $50 \mathrm{~nm}$ (blue), and $60 \mathrm{~nm}$ (green) gold NPs standards, monitored with ICPMS at $\mathrm{m} / \mathrm{z}$ 197. (C) AF4 fractograms monitored with a UV detector at $525 \mathrm{~nm}$ for $15 \mathrm{~nm}$ gold NPs labelled with OPSS-PEG-DOTA (a), OPSS-PEG-SVA (b), PEG-SH (c), OPSS-PEGSVA+AB (d), OPSS-PEG-DOTA-SVA+AB (e), OPSS-PEG-DOTA-SVA-PEG-SH (f), and OPSS-PEG-DOTA-SVA+AB-PEG-SH (g). (D) AF4 fractograms obtained for $15 \mathrm{~nm}$ gold NPs labelled with OPSS-PEG-DOTA (black), OPSS-PEG-SVA ( red), PEG-SH (blue), OPSS-PEGSVA+AB (green), OPSS-PEG-DOTA-SVA (pink), OPSS-PEG-DOTA-SVA-PEG-SH (purple), and OPSS-PEG-DOTA-SVA+AB-PEG-SH (yellow), monitored with ICPMS at m/z 197.

Table 1. Average AF4 retention times, and resulting average diameter of Au NPs $15 \mathrm{~nm}$ in diameter with different coatings, obtained using a UV detector.

\begin{tabular}{|l|c|c|}
\hline NP coating & $\begin{array}{c}\text { Retention time } \\
\text { (min) }\end{array}$ & Diameter (nm) \\
\hline (a) OPSS-PEG-DOTA & 23.2 & $42.7 \pm 0.4$ \\
\hline (b) OPSS-PEG-SVA & 22.7 & $41.3 \pm 0.4$ \\
\hline (c) PEG-SH & 17.3 & $26.3 \pm 0.3$ \\
\hline (d) OPSS-PEG-SVA-AB & 24.1 & $45.2 \pm 0.4$ \\
\hline $\begin{array}{l}\text { (e) OPSS-PEG-DOTA + } \\
\text { OPSS-PEG-SVA-AB }\end{array}$ & 24.2 & $45.5 \pm 0.4$ \\
\hline $\begin{array}{l}\text { (f) OPSS-PEG-DOTA + } \\
\text { OPSS-PEG-SVA + }\end{array}$ & 21.3 & $37.4 \pm 0.4$ \\
\hline $\begin{array}{l}\text { (g) OPSS-SH } \\
\text { OPSS-PEG-SVA-AB + } \\
\text { PEG-SH }\end{array}$ & 22.1 & $39.7 \pm 0.3$ \\
\hline
\end{tabular}




\subsubsection{Raman scattering}

Fig. 4 shows Raman spectra of Au NPs coated with OPSS-PEG-DOTA (Fig. 4A) and OPSSPEG-SVA-AB (Fig. 4B). Raman measurements demonstrated the NP labelling despite of the negligible SERS effect shown by $15 \mathrm{~nm}$ Au NPs. ${ }^{39-40}$ Fig. 4A shows the Raman spectra of OPSS-PEG-DOTA (black line) and OPSS-PEG-DOTA-coated Au NPs (red line). Both spectra show similar bands in the low-frequency region, with main bands at $365 \mathrm{~cm}^{-1}, 584 \mathrm{~cm}^{-1}$ and $718 \mathrm{~cm}^{-1}$, associated to $\delta(\mathrm{C}-\mathrm{S}-\mathrm{S}), v(\mathrm{~S}-\mathrm{S}), v_{\mathrm{ip}}(\mathrm{C}-\mathrm{S})$, respectively. ${ }^{41-42}$ Significant differences may also be observed. Band at $265 \mathrm{~cm}^{-1}$, which only appear in the spectrum of OPSS-PEGDOTA-coated Au NPs can be ascribed to the $v(\mathrm{Au}-\mathrm{S})$, which confirms NP labelling. ${ }^{43}$ Bands in the OPSS-PEG-DOTA spectrum between $840 \mathrm{~cm}^{-1}$ and $1000 \mathrm{~cm}^{-1}$ are associated to several ring modes of the pyridyl ring, and that at $775 \mathrm{~cm}^{-1}$ may be associated to normal mode with significant contribution of $v(\mathrm{C}-\mathrm{N})$, which demonstrates that OPSS binding to Au is throughout a disulphide bridge.

Labelling of OPSS-PEG with SVA and AB is shown in Figure 4B (red line). Band at $300 \mathrm{~cm}^{-}$ 1 and $633 \mathrm{~cm}^{-1}$ corresponding to the $v(\mathrm{Au}-\mathrm{S})$ and $v(\mathrm{C}-\mathrm{S})$ from $-\mathrm{CH}_{2}-\mathrm{S}-\mathrm{S}-$, respectively, indicates the labelling with OPSS, which is in good agreement with the absence of bands related to pyridine (doublet at $1450 / 1480 \mathrm{~cm}^{-1}$, associated to monosubstituted pyridine semicircle stretching, non-bonded OPSS-PEG-SVA (blue line)). The labelling with AB is demonstrated by the absence of bands associated to succinimide, i.e., bands at $278 \mathrm{~cm}^{-1}$ associated to in-plane (ip), $\delta(\mathrm{N}-\mathrm{O}), \delta(\mathrm{C}=\mathrm{O}) \mathrm{ip}, v(\mathrm{C}-\mathrm{N})$ and $\delta(\mathrm{C}-\mathrm{O})$; at $364 \mathrm{~cm}^{-1}$ associated to $v(\mathrm{C}-\mathrm{N})$ and $v(\mathrm{C}-\mathrm{C})$ and at $537 / 580 \mathrm{~cm}^{-1}$ corresponding to $\delta(\mathrm{C}=\mathrm{O})$, (blue line). Bands a 1240 $\mathrm{cm}^{-1}$ and $1670 \mathrm{~cm}^{-1}$ are typical of amide III and amide I, respectively, from the $\beta$-sheets structure of polypeptide backbone (with $\mathrm{H}$-bonds between $\mathrm{C}=\mathrm{O}$ and $\mathrm{N}-\mathrm{H}$ groups from adjacent chains arranged either in parallel or anti-parallel modes, ${ }^{44}$ in good agreement with those found in the spectrum of the non-bonded antibody (black line). The intense band at $1000 \mathrm{~cm}^{-1}$ may be associated to the breathing mode of phenylalanine (Phe) aromatic ring. Also, the band at $1550 \mathrm{~cm}^{-1}$ may be related to the $\mathrm{W} 3$ mode of tryptophan (Trp) $\left(50 \%\right.$ indole $v\left(\mathrm{C}_{2}=\mathrm{C}_{3}\right), 23 \% \mathrm{v}($ $\left.\mathrm{N}_{1}-\mathrm{C}_{2}\right)$ and $19 \% \delta\left(\mathrm{C}_{2}-\mathrm{H}\right)$ vibrations) and the weak band at $757 \mathrm{~cm}^{-1}$ may be associated to the W18 vibrational mode of Trp and Phe (cation- $\pi$ interaction), ${ }^{45}$ demonstrating the successful labelling of Au NPs with AB via OPSS-PEG-SVA linker.
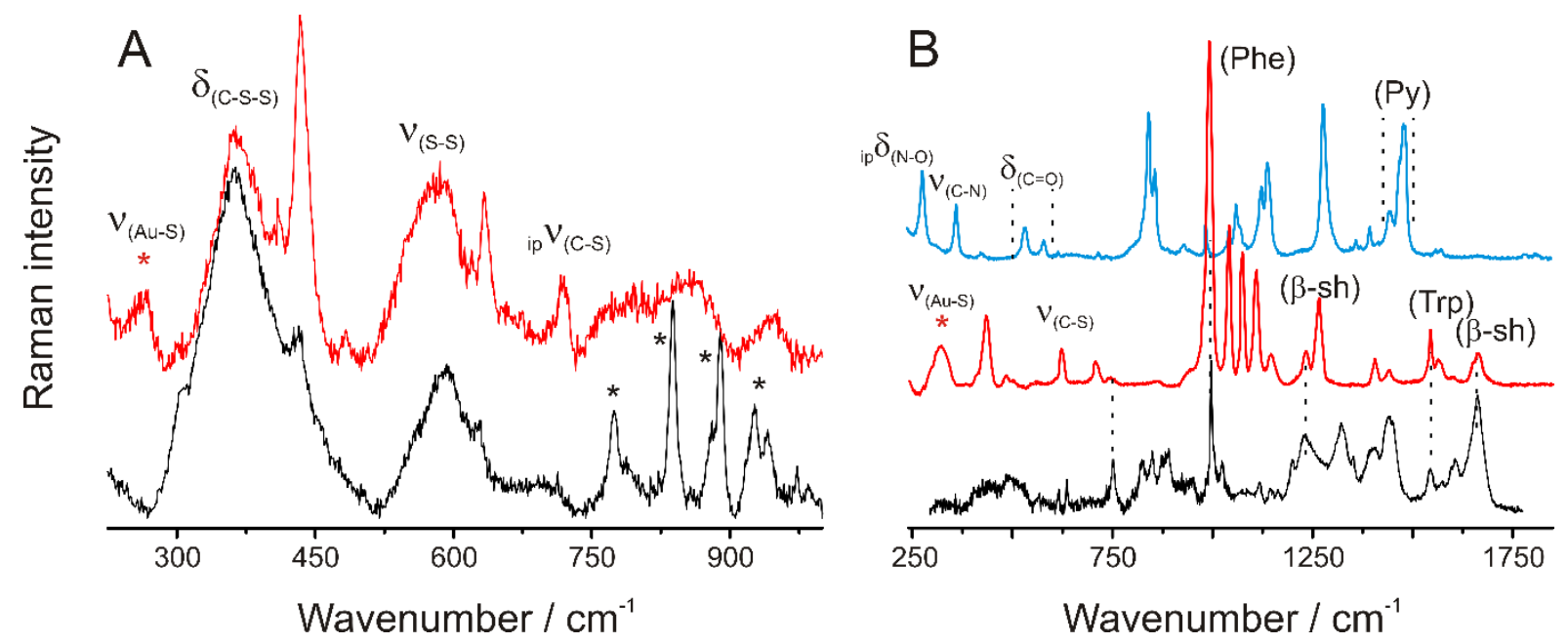
Fig. 4. Raman spectra of (A) OPSS-PEG-DOTA (black line) and $15 \mathrm{~nm}$ Au NPs coated with OPSS-PEG-DOTA (red line) and (B) AB, Erbitux (black line), $15 \mathrm{~nm}$ Au NPs coated with OPSS-PEG-SVA-AB (red line) and OPSS-PEG-SVA (blue line).

\subsubsection{Force Distance Spectroscopy}

We have used AFM force-distance spectroscopy in order to give further insight into the NPs coating process by characterising the elastic properties of the modified NPs. Fig. 5 shows forcedistance curves obtained for the mica substrate (Fig. 5A), $15 \mathrm{~nm}$ gold NPs (Fig. 5B), $15 \mathrm{~nm}$ gold NPs coated with OPSS-PEG-DOTA (Fig. 5C), $15 \mathrm{~nm}$ gold NPs coated with OPSS-PEGSVA-AB (Fig. 5D), and with the complete coating, i.e., OPSS-PEG-DOTA + OPSS-PEGSVA-AB + PEG-SH (Fig. 5E). The corresponding contact-mode AFM images are shown in the inset. The force-distance profile in Fig. 5A is typical of an uncoated cantilever and a stiff hydrophilic surface, with a linear dependence of the force on the distance in both the approach and retract regions. The hysteresis found in the point of tip-surface attachment and detachment is related to strong adhesion forces due to a few monolayers of water on both the substrate surface and the cantilever. When covered with Au NPs, all samples showed the same slope in the linear region of the force-distance curves as that found on the mica substrate, as would be expected once the NPs layer has been penetrated. However, clear differences can be found in the attachment / detachment region, both when comparing between naked mica and the NPscovered substrate, and between NPs with different surface modifications.
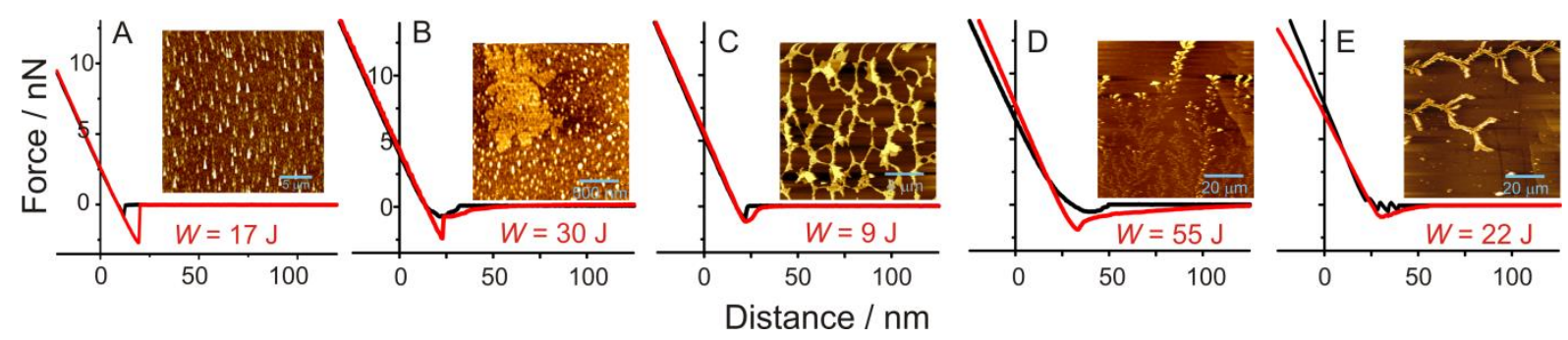

Fig. 5. AFM force-distance curves of the muscovite mica substrate (A), $15 \mathrm{~nm}$ diameter gold NPs (B), $15 \mathrm{~nm}$ gold NPs coated with DOTA (C), $15 \mathrm{~nm}$ gold NPs coated with SVA-AB (D), and $15 \mathrm{~nm}$ gold NPs coated with DOTA-SVA-AB-PEG-SH (E), in all cases deposited on the muscovite mica substrate. Black and red lines correspond to approach and retraction, respectively. Inset: corresponding contact-mode AFM images.

In the case of naked Au NPs, the attachment region in the approach curve extends over a wider region than in the case of the mica substrate without NPs, indicating that additional energy (area integrated between the force-distance curve and the zero-force line) is necessary to break through the layer of Au NPs. Similarly, the retraction curve shows that, after detaching from the mica surface (initial vertical rise similar to that in Fig. 5A), additional energy is required to detach the tip form the layer of Au NPs. This pattern is typical of capillary phenomena ${ }^{46}$ and, in this case, it may be attributed to isotropic adhesive forces between the naked NPs, which are probably covered by a few layers of water. This is in agreement with the corresponding contactmode AFM image (Fig. 5B, inset), where Au NPs appear as three-dimensional aggregates. Interestingly, in the case of gold NPs modified with OPSS-PEG-DOTA (Fig. 5C), the energy required to break through the layer of NPs (area within the black line around the point of contact), the adhesion force (minimum in the retraction curve), and the adhesion work are 
smaller than those obtained with both the clean mica substrate and with naked gold NPs. This must be due to the hydrophobic OPSS-PEG-DOTA layer preventing particle aggregation, in good agreement with the TEM images in Fig. 2B, and making it easier for the tip to break through the NP layer, as well as reducing the capillary attractive forces between the NPs and the tip. AFM contact-mode images (Fig. 5C, inset) support this assumption: DOTA-coated NPs display two-dimensional and nearly one-dimensional aggregates in a hexagonal distribution pattern matching the hexagonal symmetry of the mica substrate, ${ }^{47-49}$ an indication that NPsubstrate interactions are stronger than NP-NP interactions.

Force-distance curves for gold NPs coated with OPSS-PEG-SVA-AB (Fig. 5D) suggest an increased cohesion energy between NPs, and an increased adhesion energy of the NPs with the AFM probe, which are now higher than in the case of naked Au NPs. AFM contact-mode images (Fig. 5D, inset) show that NPs arrange themselves in a fractal pattern. Together, these observations suggest the presence of directed, anisotropic, attractive interactions between the NPs, which dominate over NP-substrate interactions. Finally, in the case of gold NPs with the complete modification (OPSS-PEG-DOTA + OPSS-PEG-SVA-AB + PEG-SH, Fig. 5E), the behaviour is intermediate between that of OPSS-PEG-DOTA- and OPSS-PEG-SVA-ABcoated NPs: the adhesion energy is intermediate, and contact-mode AFM images show an hexagonal arrangement of fractal patterns, suggesting the coexistence of anisotropic, directional interactions among NPs and similarly strong NP-substrate interactions.

These results are indirect evidence of the success of the successive modification steps, as the addition of a new component on the coating layer clearly leads to a modification of the strength and directionality of the interactions between NPs, and between the NPs and the substrate. The reproducibility of both the force-distance curves and the calculated adhesion energy suggests that AFM force-distance measurements can be used as a suitable method for characterizing NP coating.

\subsection{Radiolabelling and cell uptake}

To demonstrate the success of the screening strategy developed here, the level of binding of the screened NPs to a panel of 5 cancer cells expressing EGFR at different levels was examined. This was carried out in the absence and presence of a large excess of $\mathrm{AB}(>100 \mathrm{x}$ the amount conjugated to the $\left.\left[{ }^{177} \mathrm{Lu}\right]-\mathrm{AB}-\mathrm{NPs}\right)$ which will compete with the targeting $\mathrm{AB}$ attached to the ${ }^{177} \mathrm{Lu}-\mathrm{NPs}$. Fig. 6A shows the expression of EGFR on each cell line. The order of expression of EGFR was MDA-MB-468 >> HCT8 > > SKBr3 > HCT116 > SW620. EGFR expression by SW620 cells was below the detection limit of the assay. The uptake of $\left[{ }^{177} \mathrm{Lu}\right]-\mathrm{AB}-\mathrm{Au}$ NPs showed a similar sequence (Fig. 6B). MDA-MB-468 cells exhibited much higher uptake corresponding with their high EGFR expression. The presence of a large excess of $\mathrm{AB}$ decreased the binding of AB-targeted ${ }^{177} \mathrm{Lu}-\mathrm{NPs}$ by $95 \%$ (black) demonstrating that only about $5 \%$ of the cell binding was due to non-targeting. This indicates that the cellular delivery of the cytotoxic radionuclide $\left[{ }^{177} \mathrm{Lu}\right]$ is dependent on EGFR expression, so potentially providing effective targeted anti-cancer therapy for tumour cells over-expressing EGFR. 

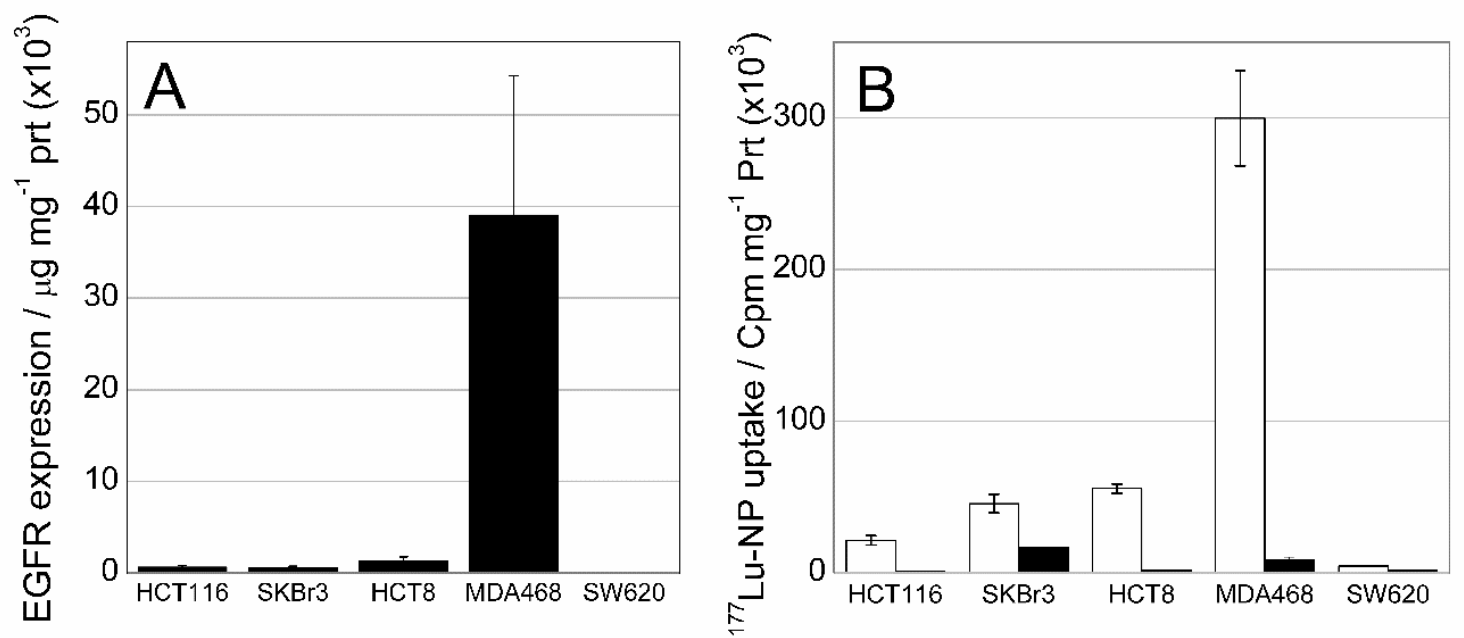

Fig. 6. EGFR-expression and ${ }^{177} \mathrm{Lu}-\mathrm{AB}-\mathrm{AuNP}$ uptake by cancer cells determined using an ELISA assay (A) and uptake of ${ }^{177} \mathrm{Lu}-\mathrm{AB}$-AuNPs determined by incubating cells with ${ }^{177} \mathrm{Lu}-$ AB-AuNPs alone (white) or in the presence of a large (100-fold excess of AB (black) to block the EGFR binding (non-receptor mediated cell binding - non-specific) (B). Units: EGFR expression - ng receptor/mg protein; ${ }^{177} \mathrm{Lu}-\mathrm{AB}-\mathrm{AuNP}$ uptake - cpm/mg protein/106 cpm incubated activity

\section{Conclusions}

Targeted nuclear medicine has the potential to accelerate, simplify and reduce costs of the implementation of personalized medicine. The purpose of fabricating EGFR-targeted ${ }^{177} \mathrm{Lu}-$ AB-NPs is to deliver ${ }^{177} \mathrm{Lu}$ specifically to cancer cells expressing EGFR at high levels. We have demonstrated that a combination of physico-chemical techniques allows to test whether the sequential functionalisation of $15 \mathrm{~nm}$ gold NPs with OPSS-PEG- linkers has been successful. TEM demonstrated that completely functionalised NPs showed good stability with no evidence of aggregation. The high sensitivity characteristic of AF4 coupled with UV-vis spectrophotometry, ICPMS and MALS detectors allowed us to calculate the diameter of the organic shell with a precision $<1 \mathrm{~nm}$. After addition of PEG-SH, the shell tends to slightly contract because of dipole-dipole interactions and H-bonding with the OPSS-PEGDOTA/SVA, leading to a final diameter of $40 \mathrm{~nm}$ (including the gold core). Raman scattering allowed to confirm the presence of DOTA and PEG-SH on the surface of the modified NPs. Finally, both AFM images and force-distance curves proved efficient tools to identify successful chemical modification of the NPs, as the organic coating strongly influences the strength of NP-NP, NP-substrate and NP-tip interactions. Based on the high reproducibility of the results, the combination of AFM imaging and force distance spectroscopy is proposed as a reliable method for the characterization of the chemical and biological modification of metal NPs. Finally, we demonstrated that Au NPs assembled as described could be radiolabelled with high $(>75 \%)$ radiolabelling efficiency and that they provide effective targeted anti-cancer therapy for tumour cells over-expressing EGFR.

\section{Supporting Information}


The Supporting Information is available free of charge on the ACS Publications website at DOI:

- AF4-ICPMS elution conditions; working principle of AF4; calibration curve and AF4 fractograms of Au NPs and mobile phase.

\section{Conflicts of interest}

There are no conflicts to declare.

\section{Acknowledgements}

Funded by Chief Scientists Office (TCS/16/07), TENOVUS Scotland (G15-03) and the University of Aberdeen Development Trust. KCN gratefully acknowledges the University of Aberdeen for the Elphinstone PhD scholarship (RG13451-10) and to Postnova Analytics UK for training and loan of the AFFF system. Microscopy was performed in the Microscopy and Histology Core Facility at the University of Aberdeen. GC acknowledges D. Zatón for useful discussions.

\section{References}

1. Baumann, M.; Krause, M.; Overgaard, J.; Debus, J.; Bentzen, S. M.; Daartz, J.; Richter, C.; Zips, D.; Bortfeld, T., Radiation Oncology in the Era of Precision Medicine. Nat. Rev. Cancer 16 (2016) 234.

2. Clarke, M. F.; Dick, J. E.; Dirks, P. B.; Eaves, C. J.; Jamieson, C. H. M.; Jones, D. L.; Visvader, J.; Weissman, I. L.; Wahl, G. M., Cancer Stem Cells-Perspectives on Current Status and Future Directions: Aacr Workshop on Cancer Stem Cells. Cancer Res. 66 (2006) 9339-44.

3. Chen, J.; Chen, Q.; Liang, C.; Yang, Z.; Zhang, L.; Yi, X.; Dong, Z.; Chao, Y.; Chen, Y.; Liu, Z., Albumin-Templated Biomineralizing Growth of Composite Nanoparticles as Smart Nano-Theranostics for Enhanced Radiotherapy of Tumors. Nanoscale 9 (2017) 14826-35.

4. Trembleau, L.; Simpson, M.; Cheyne, R. W.; Escofet, I.; Appleyard, M. V. C. A. L.; Murray, K.; Sharp, S.; Thompson, A. M.; Smith, T. A. D., Development of $18 \mathrm{f}-$ Fluorinatable Dendrons and Their Application to Cancer Cell Targeting. New J. Chem. 35 (2011) 2496-502.

5. Kobayashi, H.; Sato, N.; Saga, T.; Nakamoto, Y.; Ishimori, T.; Toyama, S.; Togashi, K.; Konishi, J.; Brechbiel, M. W., Monoclonal Antibody-Dendrimer Conjugates Enable Radiolabeling of Antibody with Markedly High Specific Activity with Minimal Loss of Immunoreactivity. Eur. J. Nucl. Med. 27 (2000) 1334-39.

6. Yordanov, A. T.; Hens, M.; Pegram, C.; Bigner, D. D.; Zalutsky, M. R., Antitenascin Antibody 81c6 Armed with 177Lu: In Vivo Comparison of Macrocyclic and Acyclic Ligands. Nucl. Med. Biol. 34 (2007) 173-83.

7. D'Huyvetter, M.; Aerts, A.; Xavier, C.; Vaneycken, I.; Devoogdt, N.; Gijs, M.; Impens, N.; Baatout, S.; Ponsard, B.; Muyldermans, S.; Caveliers, V.; Lahoutte, T., Development of 177LuNanobodies for Radioimmunotherapy of HER2-Positive Breast Cancer: Evaluation of Different Bifunctional Chelators. Contrast Media Mol. Imaging 7 (2012) 254-64.

8. Haume, K.; Rosa, S.; Grellet, S.; Śmiałek, M. A.; Butterworth, K. T.; Solov'yov, A. V.; Prise, K. M.; Golding, J.; Mason, N. J., Gold Nanoparticles for Cancer Radiotherapy: A Review. Cancer Nanotech. 7 (2016) 8.

9. Murphy, C. J.; Gole, A. M.; Stone, J. W.; Sisco, P. N.; Alkilany, A. M.; Goldsmith, E. C.; Baxter, S. C., Gold Nanoparticles in Biology: Beyond Toxicity to Cellular Imaging. Acc. Chem. Res. 41 (2008) 172130.

10. Yook, S.; Cai, Z.; Lu, Y.; Winnik, M. A.; Pignol, J.-P.; Reilly, R. M., Radiation Nanomedicine for Egfr-Positive Breast Cancer: Panitumumab-Modified Gold Nanoparticles Complexed to the B-ParticleEmitter, 177Lu. Mol. Pharm. 12 (2015) 3963-72. 
11. Chattopadhyay, N.; Cai, Z.; Pignol, J.-P.; Keller, B.; Lechtman, E.; Bendayan, R.; Reilly, R. M., Design and Characterization of HER-2-Targeted Gold Nanoparticles for Enhanced X-Radiation Treatment of Locally Advanced Breast Cancer. Mol. Pharm. 7 (2010) 2194-206.

12. Sun, X.; Zhang, G.; Patel, D.; Stephens, D.; Gobin, A. M., Targeted Cancer Therapy by Immunoconjugated Gold-Gold Sulfide Nanoparticles Using Protein $\mathrm{G}$ as a Cofactor. Ann. Biomed. Eng. 40 (2012) 2131-39.

13. lavicoli, P.; Urbán, P.; Bella, A.; Ryadnov, M. G.; Rossi, F.; Calzolai, L., Application of Asymmetric Flow Field-Flow Fractionation Hyphenations for Liposome-Antimicrobial Peptide Interaction. J. Chromatogr. A 1422 (2015) 260-69.

14. Hagendorfer, H.; Kaegi, R.; Traber, J.; Mertens, S. F. L.; Scherrers, R.; Ludwig, C.; Ulrich, A., Application of an Asymmetric Flow Field Flow Fractionation Multi-Detector Approach for Metallic Engineered Nanoparticle Characterization - Prospects and Limitations Demonstrated on $\mathrm{Au}$ Nanoparticles. Anal. Chim. Acta 706 (2011) 367-78.

15. Omar, J.; Boix, A.; Kerckhove, G.; von Holst, C., Optimisation of Asymmetric Flow Field-Flow Fractionation for the Characterisation of Nanoparticles in Coated Polydisperse TiO2 with Applications in Food and Feed. Food Addit. Contam. Part A Chem. Anal. Control Expo Risk Assess. 33 (2016) 177584.

16. Hagendorfer, H.; Kaegi, R.; Parlinska, M.; Sinnet, B.; Ludwig, C.; Ulrich, A., Characterization of Silver Nanoparticle Products Using Asymmetric Flow Field Flow Fractionation with a Multidetector Approach - a Comparison to Transmission Electron Microscopy and Batch Dynamic Light Scattering. Anal. Chem. 84 (2012) 2678-85.

17. Feldmann, J.; Raab, A.; Krupp, E. M., Importance of Icpms for Speciation Analysis Is Changing: Future Trends for Targeted and Non-Targeted Element Speciation Analysis. Anal. Bioanal.Chem. 410 (2018) 661-67.

18. Hoo, C. M.; Starostin, N.; West, P., A Comparison of Atomic Force Microscopy (AFM) and Dynamic Light Scattering (DLS) Methods to Characterize Nanoparticle Size Distributions. J. Nanopart. Res. 10 (2008) 89-96.

19. Carney, R. P.; Kim, J. Y.; Qian, H.; Jin, R.; Mehenni, H.; Stellacci, F.; Bakr, O. M., Determination of Nanoparticle Size Distribution Together with Density or Molecular Weight by 2D Analytical Ultracentrifugation. Nat. Comm. 2 (2011) 335.

20. Hassellöv, M.; Readman, J. W.; Ranville, J. F.; Tiede, K., Nanoparticle Analysis and Characterization Methodologies in Environmental Risk Assessment of Engineered Nanoparticles. Ecotoxicology 17 (2008) 344-61.

21. Grabar, K. C.; Brown, K. R.; Keating, C. D.; Stranick, S. J.; Tang, S.-L.; Natan, M. J., Nanoscale Characterization of Gold Colloid Monolayers: A Comparison of Four Techniques. Anal. Chem. 69 (1997) 471-77.

22. Baalousha, M.; Lead, J. R., Characterization of Natural and Manufactured Nanoparticles by Atomic Force Microscopy: Effect of Analysis Mode, Environment and Sample Preparation. Colloids Surf., A 419 (2013) 238-47.

23. Rao, A.; Schoenenberger, M.; Gnecco, E.; Th, G.; Meyer, E.; Brändlin, D.; Scandella, L., Characterization of Nanoparticles Using Atomic Force Microscopy. J. Phys: Conf. Ser. 61 (2007) 971.

24. Haynes, C. L.; Van Duyne, R. P., Nanosphere Lithography: A Versatile Nanofabrication Tool for Studies of Size-Dependent Nanoparticle Optics. J. Phys. Chem. B 105 (2001) 5599-611.

25. Neuman, K. C.; Nagy, A., Single-Molecule Force Spectroscopy: Optical Tweezers, Magnetic Tweezers and Atomic Force Microscopy. Nat. Methods 5 (2008) 491.

26. McNamee, C. E.; Pyo, N.; Higashitani, K., Atomic Force Microscopy Study of the Specific Adhesion between a Colloid Particle and a Living Melanoma Cell: Effect of the Charge and the Hydrophobicity of the Particle Surface. Biophys. J . 91 (2006) 1960-69.

27. Hinterdorfer, P.; Baumgartner, W.; Gruber, H. J.; Schilcher, K.; Schindler, H., Detection and Localization of Individual Antibody-Antigen Recognition Events by Atomic Force Microscopy. Proc. Natl. Acad. Sci. 93 (1996) 3477. 
28. van Buul, A. M.; Schwartz, E.; Brocorens, P.; Koepf, M.; Beljonne, D.; Maan, J. C.; Christianen, P. C. M.; Kouwer, P. H. J.; Nolte, R. J. M.; Engelkamp, H.; Blank, K.; Rowan, A. E., Stiffness Versus Architecture of Single Helical Polyisocyanopeptides. Chem. Sci. 4 (2013) 2357-63.

29. Duner, G.; Thormann, E.; Dedinaite, A.; Claesson, P. M.; Matyjaszewski, K.; Tilton, R. D., Nanomechanical Mapping of a High Curvature Polymer Brush Grafted from a Rigid Nanoparticle. Soft Matter 8 (2012) 8312-20.

30. Guo, Y.-B.; Wang, D.-G.; Zhang, S.-W., Adhesion and Friction of Nanoparticles/Polyelectrolyte Multilayer Films by Afm and Micro-Tribometer. Tribol. Int. 44 (2011) 906-15.

31. Liu, H.; Bhushan, B., Nanotribological Characterization of Molecularly Thick Lubricant Films for Applications to Mems/Nems by Afm. Ultramicroscopy 97 (2003) 321-40.

32. Yang, S.; Zhang, H.; Hsu, S. M., Correction of Random Surface Roughness on Colloidal Probes in Measuring Adhesion. Langmuir 23 (2007) 1195-202.

33. Xue, Y.; Li, X.; Li, H.; Zhang, W., Quantifying Thiol-Gold Interactions Towards the Efficient Strength Control. Nat. Comm. 5 (2014) 43-48.

34. Jokerst, J. V.; Lobovkina, T.; Zare, R. N.; Gambhir, S. S., Nanoparticle Pegylation for Imaging and Therapy. Nanomedicine 6 (2011) 715-28.

35. Rahme, K.; Chen, L.; Hobbs, R. G.; Morris, M. A.; O'Driscoll, C.; Holmes, J. D., Pegylated Gold Nanoparticles: Polymer Quantification as a Function of PEG Lengths and Nanoparticle Dimensions. RSC Adv. 3 (2013) 6085-94.

36. Pelaz, B.; del Pino, P.; Maffre, P.; Hartmann, R.; Gallego, M.; Rivera-Fernández, S.; de la Fuente, J. M.; Nienhaus, G. U.; Parak, W. J., Surface Functionalization of Nanoparticles with Polyethylene Glycol: Effects on Protein Adsorption and Cellular Uptake. ACS Nano 9 (2015) 6996-7008.

37. Yook, S.; Cai, Z.; Lu, Y.; Winnik, M. A.; Pignol, J.-P.; Reilly, R. M., Intratumorally Injected 177luLabeled Gold Nanoparticles: Gold Nanoseed Brachytherapy with Application for Neoadjuvant Treatment of Locally Advanced Breast Cancer. J. Nuc. Med. 57 (2016) 936-42.

38. Huefner, A.; Septiadi, D.; Wilts, B. D.; Patel, I. I.; Kuan, W.-L.; Fragniere, A.; Barker, R. A.; Mahajan, S., Gold Nanoparticles Explore Cells: Cellular Uptake and Their Use as Intracellular Probes. Methods 68 (2014) 354-63.

39. Njoki, P. N.; Lim, I. I. S.; Mott, D.; Park, H.-Y.; Khan, B.; Mishra, S.; Sujakumar, R.; Luo, J.; Zhong, C.-J., Size Correlation of Optical and Spectroscopic Properties for Gold Nanoparticles. J. Phys. Chem. C 111 (2007) 14664-69.

40. Huang, X.; El-Sayed, M. A., Gold Nanoparticles: Optical Properties and Implementations in Cancer Diagnosis and Photothermal Therapy. J. Adv. Res. 1 (2010) 13-28.

41. David, C.; Foley, S.; Enescu, M., Protein S-S Bridge Reduction: A Raman and Computational Study of Lysozyme Interaction with Tcep. Phys. Chem. Chem. Phys. 11 (2009) 2532-42.

42. Szafranski, C. A.; Tanner, W.; Laibinis, P. E.; Garrell, R. L., Surface-Enhanced Raman Spectroscopy of Aromatic Thiols and Disulfides on Gold Electrodes. Langmuir 14 (1998) 3570-79.

43. Turcotte, S. B.; Benner, R. E.; Riley, A. M.; Li, J.; Wadsworth, M. E.; Bodily, D., Application of Raman Spectroscopy to Metal-Sulfide Surface Analysis. Appl. Opt. 32 (1993) 935-38.

44. Wen, Z. Q., Raman Spectroscopy of Protein Pharmaceuticals. J. Pharm. Sci. 96 (2007) 2861-78.

45. Juszczak, L. J.; Desamero, R. Z. B., Extension of the Tryptophan X(2,1) Dihedral Angle - W3 Band Frequency Relationship to a Full Rotation: Correlations and Caveats. Biochemistry 48 (2009) 2777-87.

46. McConney, M. E.; Schaber, C. F.; Julian, M. D.; Barth, F. G.; Tsukruk, V. V., Viscoelastic Nanoscale Properties of Cuticle Contribute to the High-Pass Properties of Spider Vibration Receptor (Cupiennius Salei Keys). J. Royal Soc. Interface 4 (2007) 1135-43.

47. Fukuma, T.; Ueda, Y.; Yoshioka, S.; Asakawa, H., Atomic-Scale Distribution of Water Molecules at the Mica-Water Interface Visualized by Three-Dimensional Scanning Force Microscopy. Phys. Rev. Lett. 104 (2010) 016101.

48. $\quad$ Feenstra, J.; van Eerden, M.; Lemmens, A. K.; de Poel, W.; Kouwer, P. H. J.; Rowan, A. E.; Schermer, J. J., Muscovite Mica as a Growth Template of PC61BM Crystallites for Organic Photovoltaics. CrystEngComm 19 (2017) 4424-36. 
49. Small Organic Molecules on Surfaces. Springer-Verlag Berlin Heidelberg: Berlin, Germany, 2013; p XV, 323. 
Graphical Abstract

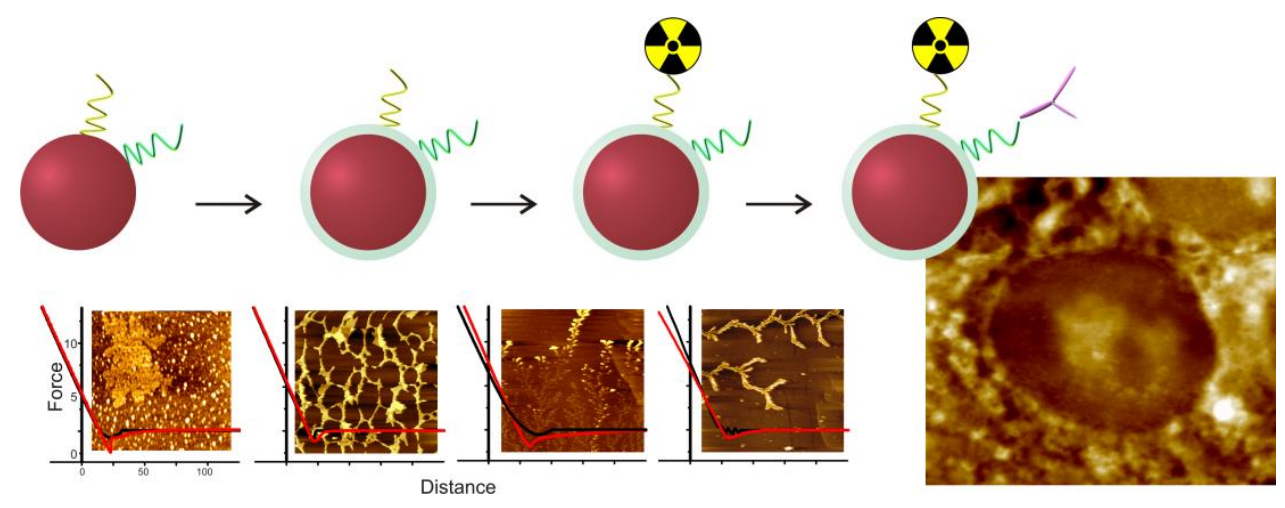

